\title{
QUASI-MATRIX LOGIC AS A PARACONSISTENT LOGIC FOR DUBITABLE INFORMATION*
}

We distinguish three types of statements - indubitably true statements (the value " $n$ "), indubitably false statements (the value "i") and statements of dubitable information (the value "c").

A set $\left(Q, G, g f_{1}, \ldots, g f_{s}\right)$ is a quasi-matrix. $Q$ and $G$ are non-empty sets. $G \subset Q . g f_{1}, \ldots, g f_{s}$ are quasi-functions. If a function is a correspondence in virtue of which an object from some (function) domain is related with a certain object (from the range of the function) then the quasi-function is a correspondence in virtue of which an object from some domain is related with some object from a certain subset of some set (from the range of the quasi-function).

Examples. Function: $\{(a, d),(b, k),(c, k)\}$. Quasi-functions: $\{(a, d) \underline{\vee}(a, k)$, $(c, m)\}=\{\{(a, d),(c, m)\},\{(a, k),(c, m)\}\} ;\left\{\underline{\vee}_{4}((a, k),(a, n),(c, k),(c, n))\right.$, $(d, r)\}=\{\{(a, k),(d, r)\},\{(a, n),(d, r)\},\{(c, k),(d, r)\},\{(c, n),(d, r)\}\} . \underline{\vee}$ and $\underline{\vee}_{4}$ are two and four-places strong disjunction (in metalanguage) respectively. We can say: a quasi-function is a set of sets of functions.

The language has the symbols $T, K, \neg, \wedge, \vee, \supset$ that are defined through the following tables:

${ }^{*}$ This paper is supported by INTAS, grant 95-0365. 


\begin{tabular}{c|c|c|c}
$A$ & $\neg A$ & $\mathrm{~T} A$ & $\mathrm{~K} A$ \\
\hline $\mathrm{n}$ & $\mathrm{i}$ & $\mathrm{n}$ & $\mathrm{n}$ \\
$\mathrm{c}$ & $\mathrm{c}$ & $\mathrm{i}$ & $\mathrm{n}$ \\
$\mathrm{i}$ & $\mathrm{n}$ & $\mathrm{i}$ & $\mathrm{i}$
\end{tabular}

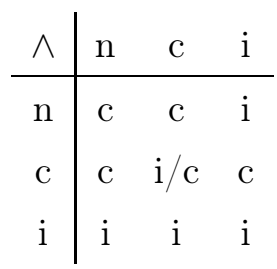

\begin{tabular}{c|ccc}
$\vee$ & $\mathrm{n}$ & $\mathrm{c}$ & $\mathrm{i}$ \\
\hline $\mathrm{n}$ & $\mathrm{n}$ & $\mathrm{n}$ & $\mathrm{n}$ \\
$\mathrm{n}$ & $\mathrm{n}$ & $\mathrm{n} / \mathrm{c}$ & $\mathrm{i}$ \\
$\mathrm{i}$ & $\mathrm{n}$ & $\mathrm{c}$ & $\mathrm{i}$
\end{tabular}

\begin{tabular}{c|ccc}
$\supset$ & $\mathrm{n}$ & $\mathrm{c}$ & $\mathrm{i}$ \\
\hline $\mathrm{n}$ & $\mathrm{n}$ & $\mathrm{c}$ & $\mathrm{i}$ \\
$\mathrm{n}$ & $\mathrm{n}$ & $\mathrm{n} / \mathrm{c}$ & $\mathrm{c}$ \\
$\mathrm{i}$ & $\mathrm{n}$ & $\mathrm{n}$ & $\mathrm{n}$
\end{tabular}

$\mathrm{i} / \mathrm{c}$ and $\mathrm{n} / \mathrm{c}$ mean "either $\mathrm{i}$, or $\mathrm{c}$ " and "either $\mathrm{n}$, or $\mathrm{c}$ " respectively. $\mathrm{T}$ and $\mathrm{K}$ are symbols for such expressions "we indubitably know that ..." and "we know that ..." respectively. $\mathrm{n}$ is a designated value.

The calculus that formalises the described semantics contains the axiomschemes that coincide with the axiom-schemes of classical propositional calculus. In these axiom-schemes metavariables denote modalised formulas. (The modalised formula definition: if $A$ is a formula of classical propositional calculus, then $\mathrm{T} A$ and $\mathrm{K} A$ are modalised formulas; if $B$ and $C$ are modalised formulas, then T $B, \mathrm{~K} B, \neg B,(B \wedge C),(B \vee C),(B \supset C)$ are modalised formulas; nothing else is a modalised formula.)

Except these schemes there are 29 more axiom-schemes the metavariables of which denote any formulas of the system:

$$
\begin{aligned}
\mathrm{T} A & \supset \mathrm{K} A \\
\mathrm{~T} A & \supset A \\
\neg A & \supset \neg \mathrm{T} A \\
A & \supset \mathrm{K} A \\
\neg \mathrm{K} A & \supset \neg A \\
\neg \mathrm{T} \neg A & \supset \mathrm{K} A \\
\mathrm{~K} A & \supset \neg \mathrm{T} \neg A \\
\mathrm{~T} A & \supset \mathrm{T} \mathrm{T} A \\
\mathrm{KT} A & \supset \mathrm{T} A \\
\mathrm{~K} A & \supset \mathrm{T} \mathrm{K} A \\
\mathrm{KK} A & \supset \mathrm{K} A \\
\neg \mathrm{K} A & \supset \mathrm{T}(A \supset B)
\end{aligned}
$$


QUASI-MATRIX LOGIC AS A PARACONSISTENT LOGIC ...

$$
\begin{aligned}
\mathrm{T} B & \supset \mathrm{T}(A \supset B) \\
\mathrm{T}(A \supset B) & \supset(\mathrm{T} A \supset \mathrm{T} B) \\
\mathrm{T}(A \supset B) & \supset(\mathrm{K} A \supset \mathrm{K} B) \\
\mathrm{K}(A \supset B) & \supset(\mathrm{T} A \supset \mathrm{K} B) \\
\mathrm{K} B & \supset \mathrm{K}(A \supset B) \\
\mathrm{K} \neg A & \supset \mathrm{K}(A \supset B) \\
\mathrm{T} A \wedge \mathrm{T} B & \supset \mathrm{T}(A \wedge B) \\
\mathrm{K} A \wedge \mathrm{T} B & \supset \mathrm{K}(A \wedge B) \\
\mathrm{T} A \wedge \mathrm{K} B & \supset \mathrm{K}(A \wedge B) \\
\mathrm{T}(A \wedge B) & \supset \mathrm{T} A \wedge \mathrm{T} B \\
\mathrm{~K}(A \wedge B) & \supset \mathrm{K} A \wedge \mathrm{K} B \\
\mathrm{~T} A \vee(\mathrm{K} A & \wedge K \neg A) \vee \neg \mathrm{K} A \\
\mathrm{~T} A \vee \mathrm{T} B & \supset \mathrm{T}(A \vee B) \\
\mathrm{K} A \vee \mathrm{K} B & \supset \mathrm{K}(A \vee B) \\
\mathrm{T}(A \vee B) & \supset \mathrm{T} A \vee \mathrm{K} B \\
\mathrm{~T}(A \vee B) & \supset \mathrm{K} A \vee \mathrm{T} B \\
\mathrm{~K}(A \vee B) & \supset \mathrm{K} A \vee \mathrm{K} B
\end{aligned}
$$

The rules of inference are as follows: modus ponens; the rule of substitution of any number of occurrences of $\neg \neg A$ by $A$ and vice versa; Gödel's rule

$$
A \Rightarrow \mathrm{T} A
$$

The proof definition is usual.

An inference is a non-empty finite sequence of formulas each of which is either a hypothesis from some set of hypotheses $\Gamma$, or a theorem, or a formula obtained from the preceding formulas of the sequence by one of the rules of inference including Gödel's rule. An inference is an inference of the last formula from the sequence from the hypotheses set $\Gamma$.

All the derived rules of inference of classical propositional calculus are rules of inference of the given calculus with the following restrictions: they can be applied only to modalised formulas. Indirect rules such as the rule of deduction

$$
\frac{\Gamma, A \Rightarrow B}{\Gamma \Rightarrow A \supset B}
$$


and the rule of reduction ad absurdum

$$
\frac{\Gamma, A \Rightarrow B ; \quad \Gamma, A \Rightarrow \neg B}{\Gamma \Rightarrow \neg A}
$$

can not be applied to non-modalised formulas. However, the so-called weakened rule of reduction ad absurdum

$$
\frac{\Gamma, A \Rightarrow B ; \quad \Gamma, A \Rightarrow \neg B}{\Gamma \Rightarrow \mathrm{K} \neg A}
$$

can be applied to non-modalised formulas.

In order to prove the metatheorem of semantic completeness the notion of alternative interpretation is introduced. An interpretation is a quasifunction || . The alternative interpretation is a function \lfloor\rfloor assigning a "non-fractional" value to a alternative interpretations: $\lfloor A\rfloor_{1}=n,\lfloor A\rfloor_{2}=c$. We shall present only some induction step cases from the inductive definition of the function:

$$
\begin{gathered}
\lfloor\mathrm{T} A\rfloor=\mathrm{n} \text { iff }\lfloor A\rfloor=\mathrm{n} ; \\
\lfloor\mathrm{T} A\rfloor=\mathrm{i} \text { iff }\lfloor A\rfloor=\mathrm{c} \text { or }\lfloor A\rfloor=\mathrm{i} ; \\
\lfloor\mathrm{K} A\rfloor=\mathrm{i} \text { iff }\lfloor A\rfloor=\mathrm{i} ; \\
\lfloor\mathrm{K} A\rfloor=\mathrm{n} \text { iff }\lfloor A\rfloor=\mathrm{n} \text { or }\lfloor A\rfloor=\mathrm{c} ; \\
\lfloor A \wedge B\rfloor=\mathrm{n} \text { iff }\lfloor A\rfloor=\lfloor B\rfloor=\mathrm{n} ; \\
\text { if }(\lfloor A\rfloor=\mathrm{n} \text { and }\lfloor B\rfloor=\mathrm{c}) \text { or }(\lfloor A\rfloor=\mathrm{c} \text { and }\lfloor B\rfloor=\mathrm{n}), \text { then }\lfloor A \wedge B\rfloor=\mathrm{c} ; \\
\text { if }\lfloor A\rfloor=\mathrm{i} \text { or }\lfloor B\rfloor=\mathrm{i} \text {, then }\lfloor A \wedge B\rfloor=\mathrm{i} ; \\
\text { if }\lfloor A\rfloor=\lfloor B\rfloor=\mathrm{c} \text {, then }\lfloor A \wedge B\rfloor \in\{\mathrm{i}, \mathrm{c}\} .
\end{gathered}
$$

In order to prove the metatheorem of semantic completeness of the calculus the following statement is proved: a set of formulas consistent with the calculus can be extended to a maximal consistent set.

Then the following lemma is proved: let $W$ be a set of formulas consistent with the calculus. Then there is alternative interpretation assigning a designated value to every formula from $W$.

To prove the lemma we introduce the function \lfloor\rfloor$_{W}$ that has the following feature: it is true for any arbitrary formula $A$ that $\lfloor A\rfloor_{W}=\mathrm{n}$ iff $\mathrm{T} A \in W$; $\lfloor A\rfloor_{W}=\mathrm{i}$ iff $\neg \mathrm{K} A \in W ;\lfloor A\rfloor_{W}=\mathrm{c}$ iff $\mathrm{K} A \in W$ and $\mathrm{K} \neg A \in W$.

It is proved by induction on the number of occurrences of logical terms in the formula $A$ that the function $\rfloor_{W}$ has all the properties of alternative interpretation, i.e. is alternative interpretation. 
It is possible to construct a paraconsistent logic for statements of indubitable information.

Look at the classical logic principles, the principles of constructed logic and the principles of relevant logic and dual of Hao Wang logic.

\begin{tabular}{|c|c|c|c|}
\hline Classical logic & This & Relevant & $\begin{array}{c}\text { Dual of Hao } \\
\text { Wang }\end{array}$ \\
\hline $\begin{array}{l}\text { (1) the principle of bivalency } \\
\text { (propositions take values from the } \\
\text { range }\{\mathrm{t} \text { (truth), } \mathrm{f} \text { (falsity) }\} \text { ) }\end{array}$ & $\begin{array}{l}\text { generalisation } \\
\{\mathrm{n}, \mathrm{c}, \mathrm{i}\}\end{array}$ & $\begin{array}{l}\text { generalisation } \\
\{\mathrm{n}, \mathrm{c}, \mathrm{i}\}\end{array}$ & $\begin{array}{l}\text { generalisation } \\
\{\mathrm{n}, \mathrm{c}, \mathrm{i}\}\end{array}$ \\
\hline $\begin{array}{l}\text { (2) the principle of consistency (a } \\
\text { proposition can not have both } \\
\text { values) }\end{array}$ & $\begin{array}{l}\text { can not have } \\
\text { two or three }\end{array}$ & $\begin{array}{l}\text { can have two } \\
\text { or three }\end{array}$ & $\begin{array}{l}\text { can have two } \\
\text { or three }\end{array}$ \\
\hline $\begin{array}{l}\text { (3) the principle of excluded middle } \\
\text { (a proposition necessarily has some } \\
\text { of these values) }\end{array}$ & $\begin{array}{l}\text { excluded } \\
\text { fourth }\end{array}$ & - & $\begin{array}{l}\text { excluded } \\
\text { fourth }\end{array}$ \\
\hline $\begin{array}{l}\text { (4) the principle of identity (in a } \\
\text { complex proposition, a system of } \\
\text { propositions, an argument one and } \\
\text { the same proposition has one and } \\
\text { the same value) }\end{array}$ & $\begin{array}{l}\text { the principle } \\
\text { of identity }\end{array}$ & - & - \\
\hline $\begin{array}{l}\text { (5) the principle of specifying the } \\
\text { truth value of a complex proposition } \\
\text { by truth values of elementary } \\
\text { propositions constituting it (in } \\
\text { propositional logic this principle } \\
\text { acts as a matrix principle - logical } \\
\text { connectives are defined by matrices, } \\
\text { in predicate logic it shows up in the } \\
\text { interpretation of logical terms and } \\
\text { predicates as truth functions) }\end{array}$ & $\begin{array}{l}\text { the quasi- } \\
\text { matrix } \\
\text { principle }\end{array}$ & $\begin{array}{l}\text { the quasi- } \\
\text { matrix } \\
\text { principle }\end{array}$ & $\begin{array}{l}\text { the quasi- } \\
\text { matrix } \\
\text { principle }\end{array}$ \\
\hline
\end{tabular}

State descriptions for classical logic: $\left\{a_{1}^{\prime}, \ldots, a_{r}^{\prime}, \ldots\right\}, a_{m}^{\prime}$ is $a_{m}$ or $\neg a_{m}$. State descriptions for relevant logic are subsets of the set $\left\{a_{1}^{n}, a_{1}^{c}, a_{1}^{i}, \ldots, a_{r}^{n}\right.$, $\left.a_{r}^{c}, a_{r}^{i}, \ldots\right\}$. If $\alpha$ is state description for relevant logic, then $a_{m}$ has value $\mathrm{n}$ in $\alpha$ iff $a_{m}^{n} \in \alpha, a_{m}$ has value $\mathrm{c}$ iff $a_{m}^{c} \in \alpha, a_{m}$ has value c iff $a_{m}^{i} \in \alpha$. We introduce a relevant implication: $\vDash A \rightarrow B \Longleftrightarrow A \vDash B \Longleftrightarrow$ information of $B$ about all state descriptions $(I(B, M))$ is part of information of $A$ about all state descriptions $(I(A, M)) \Longleftrightarrow M_{A} \subseteq M_{B}$. ( $M_{A}$ and $M_{B}$ are all state descriptions in which $A$, respectively $B$, has value $\mathrm{n}$. State descriptions for dual of Hao Wang logic are all subsets of the set $\left\{a_{1}^{n}, a_{1}^{c}, a_{1}^{i}, \ldots, a_{r}^{n}, a_{r}^{c}, a_{r}^{i}, \ldots\right\}$, for 
which are true: $\forall^{\prime} a_{s}\left(a_{s}^{n} \in \alpha \vee^{\prime} a_{s}^{c} \in \alpha \vee^{\prime} a_{s}^{i} \in \alpha\right) . \forall^{\prime}$ and $\vee^{\prime}$ are symbols of metalanguage.

The calculus that formalises the described semantics of relevant logic contains the axiom-schemes that coincide with the axiom-schemes of propositional calculus E.In these axiom-schemes metavariables denote modalised formulas. Except these schemes there are 28 more axiom-schemes the metavariables of which denote any formulas of the system:

$$
\begin{aligned}
\mathrm{T} A & \rightarrow \mathrm{K} A \\
\mathrm{~T} A & \rightarrow A \\
\neg A & \rightarrow \neg \mathrm{T} A \\
A & \rightarrow \mathrm{K} A \\
\neg \mathrm{K} A & \rightarrow \neg A \\
\neg \mathrm{T} \neg A & \rightarrow \mathrm{K} A \\
\mathrm{~K} A & \rightarrow \neg \mathrm{T} \neg A \\
\mathrm{~T} A & \rightarrow \mathrm{T} \mathrm{T} A \\
\mathrm{~K} \mathrm{~T} A & \rightarrow \mathrm{T} A \\
\mathrm{~K} A & \rightarrow \mathrm{T} \mathrm{K} A \\
\mathrm{~K} \mathrm{~K} A & \rightarrow \mathrm{K} A \\
\neg \mathrm{K} A & \rightarrow \mathrm{T}(A \supset B) \\
\mathrm{T} B & \rightarrow \mathrm{T}(A \supset B) \\
\mathrm{T}(A \supset B) & \rightarrow(\mathrm{T} A \supset \mathrm{T} B) \\
\mathrm{T}(A \supset B) & \rightarrow(\mathrm{K} A \supset \mathrm{K} B) \\
\mathrm{K}(A \supset B) & \rightarrow(\mathrm{T} A \supset \mathrm{K} B) \\
\mathrm{K} B & \rightarrow \mathrm{K}(A \supset B) \\
\mathrm{K} \neg A & \rightarrow \mathrm{K}(A \supset B) \\
\mathrm{T} A \wedge \mathrm{T} B & \rightarrow \mathrm{T}(A \wedge B) \\
\mathrm{K} A \wedge \mathrm{T} B & \rightarrow \mathrm{K}(A \wedge B) \\
\mathrm{T} A \wedge \mathrm{K} B & \rightarrow \mathrm{K}(A \wedge B) \\
\mathrm{T}(A \wedge B) & \rightarrow \mathrm{T} A \wedge \mathrm{T} B \\
\mathrm{~K}(A \wedge B) & \rightarrow \mathrm{K} A \wedge \mathrm{K} B \\
\mathrm{~T} A \vee \mathrm{T} B & \rightarrow \mathrm{T}(A \vee B) \\
\mathrm{K} A \vee \mathrm{K} B & \rightarrow \mathrm{K}(A \vee B) \\
\mathrm{T}(A \vee B) & \rightarrow \mathrm{T} A \vee \mathrm{K} B
\end{aligned}
$$




$$
\begin{aligned}
\mathrm{T}(A \vee B) & \rightarrow \mathrm{K} A \vee \mathrm{T} B \\
\mathrm{~K}(A \vee B) & \rightarrow \mathrm{K} A \vee \mathrm{K} B
\end{aligned}
$$

The rules of inference are as follows: modus ponens $A \rightarrow B, A \Rightarrow B$; introduction of conjunction $A, B \Rightarrow A \wedge B$; the rule of substitution of any number of occurrences of $\neg \neg A$ by $A$ and vice versa; Gödel's rule.

Dual of Hao Wang logic also includes these 28 axiom-schemes (here $\rightarrow-$ Hao Wang logic implication) and the axiom-scheme

$$
\mathrm{T} A \vee(\mathrm{K} A \wedge \mathrm{K} \neg A) \vee \neg \mathrm{K} A .
$$

\section{References}

[1] Ivlev, Yu. V., "A semantics for modal calculus", Bulletin of the Section of Logic, Vol. 17, No 3/4, 1988, pp. 114-126.

[2] Ivlev, Yu. V., "Quasi-matrix logic as a para-consistent logic for dubitable information", in Stanisław Jaśkowski Memorial Symposium "Parainconsistent Logic, Logical Philosophy, Mathematics \& Informatics", Toruń, 1998, pp. 68-69.

[3] Ivlev, Yu. V., "Semantics of restricted state-descriptions for quasi-matrix logic", Bulletin of the Section of Logic, Vol. 27, No 1/2, 1998, pp. 74-76.

[4] Voishvillo, E., "A theory of logical relevance", Logique et Analyse, 155-156 (1996), 207-228.

Yu. V. IVLEV

Faculty of Philosophy

Department of Logic

Moscow State University

Vorobjevy Gory

119899 Moscow, Russia

ivlev@log.philos.msu.su 